\title{
Laparoscopic D2 total gastrectomy and en-mass splenectomy and distal pancreatectomy for locally advanced proximal gastric cancer
}

\author{
Bin Chet $\operatorname{Toh}^{1}$ (D) Jaideepraj Rao ${ }^{1}$
}

Received: 31 March 2017 / Accepted: 28 July 2017 / Published online: 25 August 2017

(C) Springer Science+Business Media, LLC 2017

\begin{abstract}
Background Safety and efficacy of laparoscopy surgery in locally Advanced Gastric Cancers (AGC) have not been proven by randomized control trials. Therefore, standard of care for AGC is still open surgery. Here, we are presenting a 64-year-old female with proximal gastric adenocarcinoma (close to cardio-oesophageal junction) adherent to tail of pancreas, who underwent D2 total gastrectomy enmass distal pancreatectomy and splenectomy.

Methods Five ports are entered into the peritoneal cavity (Three 10-12 $\mathrm{mm}$ and two $5 \mathrm{~mm}$ ports). Another $5 \mathrm{~mm}$ stab incision is made in the epigastrium for Nathanson Liver retractor. Standard D2 Gastrectomy was performed with en-mass removal of the spleen and body and tail of the pancreas. Roux-en-Y oesophago-jejunostomy (Hand sewn) and Jejuno-Jejunostomy reconstruction were performed laparoscopically. Hereby, we present a video of the above procedure.

Results Total Operating time was 235 min. Post-operatively the patient was able to mobilize independently. Total
\end{abstract}

Parenteral Nutrition (TPN) was started and continued until post-operative day (POD) 7 once gastrografin test and blue dye test both showed no anastomotic leak. Patient started on oral feeding and was discharged home well on POD 9. Histology showed poorly differentiated adenocarcinoma with pT3N3b (17 nodes out of 62 positive).

Conclusion Although laparoscopic D2 gastrectomy and en-mass distal pancreatectomy and splenectomy are feasible and safe in advanced gastric carcinoma, its oncological value has yet to be determined.

Keywords Advanced laparoscopy · Total gastrectomy · Splenectomy · Distal pancreatectomy

\section{Compliance with ethical standards}

Disclosures Bin Chet Toh and Jaideepraj Rao have no conflicts of interest or financial ties to disclose.
Electronic supplementary material The online version of this article (doi:10.1007/s00464-017-5801-9) contains supplementary material, which is available to authorized users.

\section{Bin Chet Toh}

binchet626@yahoo.com

1 Upper Gastrointestinal and Laparoscopic Unit, General Surgery Department, Tan Tock Seng Hospital, Singapore, Singapore 